\title{
SOME TORSION-FREE GROUP RINGS WITH NILPOTENT \\ PRIME RADICALS
}

\section{KENG-TEH TAN}

(Received 17 January 1973)

Communicated by G. E. Wall

\section{Introduction}

Let $R$ be a ring with identity. We will use $J(R)$ and $P(R)$ to denote the Jacobson and prime radicals of $R$, respectively. If $G$ is a group, the group ring of $G$ over $R$ will be denoted by $R G$.

In this paper, we will prove the following results:

THEOREM 1. If $R$ is a left Goldie ring (with identity) and $G$ the infinite cyclic group, then $P(R G)$ is nilpotent and

$$
P(R G)=J(R G)=P(R) G=N G,
$$

where $N=J(R[X]) \cap R$.

TheOREM 2. If $R$ is left Noetherian and $G$ is torsion-free abelian, then $P(R G)$ is nilpotent and $P(R G)=P(R) G$.

The first result is an analogue of a theorem of Amitsur on polynomial rings [1, page 358, Theorem 1]:

If $R$ is a ring, then $J(R[X])=N[X]$, where $N=J(R[X]) \cap R$ is nil. Furthermore, $N$ contains the locally nilpotent radical $\sigma(R)$ of $R$, that is, the largest ideal of $R$ whose finitely generated subrings are nilpotent.

In fact, we will make use of this result to prove Theorem 1.

\section{Proof of the theorems}

In this section, unless otherwise stated, $R$ will always denote a left Goldie ring and $G$ the infinite cyclic group.

LEMMA 1. $P(R)=\sigma(R)=N$.

Proof. By the result of Amitsur quoted above, $\sigma(R) \subseteq N$ and $N$ is nil, hence $N$ is nilpotent, since $R$ is left Goldie. Trivially, every finitely generated subring of $N$ is nilpotent, thus $\sigma(R)=N$ by the maximality of $\sigma(R)$ with respect to the 
property that finitely generated subrings be nilpotent. Similarly, $P(R)$ is nil, so it is nilpotent and one infers in like manner that $P(R)=\sigma(R)=N$. This proves Lemma 1.

LeMMA 2. $J(R G)=N G$.

Proof: Since $N$ is nilpotent, so is $N G$. Therefore $N G \subseteq J(R G)$.

To show the reverse inclusion, let $a \in J(R G), a \neq 0$. Let $g$ be a generator of G. Then

$$
a=g^{n} f(g)
$$

for some integer $n$ and for some $f(g) \in R[g]$, the polynomial ring of $g$ considered as a variable over $R$, and the constant term of $f(g)$ is not zero. Then

$$
g f(g) \in J(R G) \text {. }
$$

We want to show that $g f(g) \in J(R[g])$. To this end, it suffices to show that $g f(g) R[g]$ is a right quasi regular right ideal of $R[g]$. Let $h(g) \in R[g]$, then

$$
g f(g) h(g) \in J(R G) \text {. }
$$

Hence there exists $g^{r} k(g) \in R G$ such that

$$
g f(g) h(g)+g^{r} k(g)+g f(g) g^{r} k(g)=0
$$

where $r$ is an integer and $k(g) \in R[g]$ has non-zero constant term. We claim that $r \geqq 0$. For if $r<0$, then

$$
g^{1-r} f(g) h(g)+k(g)+g f(g) k(g)=0 .
$$

It would follow that the constant term of $k(g)$ is zero, a contradiction. Thus

$$
g^{r} k(g) \in R[g] .
$$

This shows that $g f(g) R[g]$ is right quasi regular, so it is contained in $J(R[g])$ $=N[g]$. It follows that the coefficients of $g f(g)$ and hence of $f(g)$ are in $N$. This proves the reverse inclusion.

We remark that $J(R G) \subseteq N G$ is always valid for any ring with identity.

LEMMA 3. $P(R G)=P(R) G=N G$.

Proof: Since $P(R)$ is nilpotent, so is $P(R) G$. Thus $P(R) G \subseteq P(R G) \subseteq J(R G)$ $=N G=P(R) G$ by Lemma 2 and Lemma 1 . Hence they are all equal.

This also completes the proof of Theorem 1 .

We now prove Theorem 2. Let $R$ be left Noetherian (with identity) and $G$ be torsion-free abelian.

We first note that $P(R)$ is nilpotent, since $R$ is left Noetherian. Hence $P(R) G$ is nilpotent, thus

$$
P(R) G \subseteq P(R G)
$$


We now assume that $G$ is free abelian of finite rank $n$. If $n=1$, then we have nothing to prove by Theorem 1 . Let $n>1$. Assume that the assertion is valid for all free abelian groups of ranks less than $n$. Let $G=H K$, where $H$ is free abelian of rank $n-1$ and $K$ is infinite cyclic. Then $R H$ is Noetherian, hence

$$
P[(R H) K]=P(R H) K .
$$

Also, by induction hypothesis,

$$
P(R H)=P(R) H .
$$

However, since

we have

$$
R G=R(H K)=(R H) K,
$$

$$
\begin{aligned}
P(R G) & =P[(R H) K] \\
& =[P(R H)] K=[P(R) H] K=P(R)(H K)=P(R) G .
\end{aligned}
$$

This proves the assertion for the case $G$ is of finite rank.

Now let $G$ be arbitrary. We are left to prove that $P(R G) \subseteq P(R) G$. Let $x \in P(R G)$. Write

$$
x=x_{1} g_{1}+\cdots+x_{n} g_{n}
$$

where $x_{i} \neq 0$ for all $i$ and $g_{i} \neq g_{j}$ for $i \neq j$. Let $G_{0}$ be the subgroup of $G$ generated by $g_{1}, \cdots, g_{n}$. Then $G_{0}$ is free abelian of finite rank and $x \in R G_{0}$. By the previous paragraph,

$$
P\left(R G_{0}\right)=P(R) G_{0} .
$$

However, $x \in P(R G)$ implies that $x$ is strongly nilpotent in $R G$, in particular, $x$ is strongly nilpotent in $R G_{0}$ ([3], page 55). Hence $x \in P\left(R G_{0}\right)$. Thus $x \in P(R) G_{0}$ $\subseteq P(R) G$. This completes the proof of Theorem 2 .

\section{Some related questions}

It is not known to the author whether Theorem 2 is true for left Goldie rings. The answer would be affirmative if we know that $R$ is left Goldie and $G$ is infinite cyclic imply that $R G$ is left Goldie.

In the sequel, unless otherwise stated, $R$ will denote an arbitrary ring with identity, $G$ the infinite cyclic group, $M=J(R G) \cap R$ and $N=J(R[X]) \cap R$.

As we noted before, $J(R G) \subseteq N G$. Since $N$ is nil, so $N \subseteq J(R)$, it follows that $N G \subseteq J(R) G$. Trivially, $M \subseteq J(R G)$, thus $M G \subseteq J(R G)$. In summary, we have

PROPOSITION 4. $M G \subseteq J(R G) \subseteq N G \subseteq J(R) G$. 
COROLlaRY 5. If $J(R)=(0)$ and $G$ is torsion-free abelian, then $J(R G)=(0)$.

ProOF: We may assume that $G$ is finitely generated. Then $G$ is of finite rank, say $n$. If $n=1$, then the claim is obvious by Proposition 4. We may now complete the proof by induction on $n$.

We would like to know when will

$$
M G=J(R G)=N G .
$$

We note that if $J(R G)=N G$, then $N \subseteq J(R G)$. Thus

$$
N=N \cap R \subseteq J(R G) \cap R=M .
$$

Hence $M=N$ and so $M G=J(R G)=N G$. We further note that if $N=\sigma(R)$, then $N G$ is nil, therefore $N G \subseteq J(R G)$, and so $M G=J(R G)=N G$. For instance if $R$ is commutative or left Goldie, then $\sigma(R)=N$. Thus we have proved the following interesting

PRoposition 6. If $R$ is commutative or left Goldie, then $M G=J(R G)=N G$.

The author is unaware of any ring for which the three ideals above are not identical.

We now consider an example. Let $p$ be a fixed prime and $R$ the ring of all rational numbers whose denominators are not divisible by $p$. Then $J(R)$ $=p R \neq(0)$, since $p R$ is the set of all non-units of $R$ and it forms the unique maximal ideal of $R$. However, $J(R[X])=(0)$, so $N=(0)$. Thus, $(0)=N G \underset{\neq}{\subsetneq} J(R) G$.

\section{Acknowledgements}

This paper arises from the author's Ph.D. thesis at Queen's University at Kingston, Canada. The author wishes to express his sincere thanks to Professor I. Hughes for his guidance and encouragements during the preparation of the thesis.

\section{References}

[1] S. A. Amistur, 'Radicals of Polynomial Rings', Can. J. Math. 8 (1956), 355-361.

[2] I. N. Herstein, Noncommutative Rings (The Carus Mathematical Monographs, 1968).

[3] J. Lambek, Lectures on Rings and Modules (Blaisdell, Waltham, 1966).

Department of Mathematics

University of Malaya

Kuala Lumpur

Malaysia 